\title{
Chiral symmetry and light resonances in hot and dense matter
}

\author{
D. Cabrera, D. Fernández-Fraile, A. Gómez Nicola ${ }^{a}$ \\ Departamento de Física Teórica II, Univ. Complutense, 28040 Madrid, Spain
}

Received: 14 August 2008 / Published online: 16 December 2008

(C) Springer-Verlag / Società Italiana di Fisica 2008

\begin{abstract}
We present a study of the $\pi \pi$ scattering amplitude in the $\sigma$ and $\rho$ channels at finite temperature and nuclear density within a chiral unitary framework. Meson resonances are dynamically generated in our approach, which allows us to analyze the behavior of their associated scattering poles when the system is driven towards chiral-symmetry restoration. Medium effects are incorporated in three ways: (a) by thermal corrections of the unitarized scattering amplitudes, (b) by finite nuclear-density effects associated to a renormalization of the pion decay constant, and complementarily (c) by extending our calculation of the scalar-isoscalar channel to account for finite nuclear-density and temperature effects in a microscopic many-body implementation of pion dynamics. Our results are discussed in connection with several phenomenological aspects relevant for nuclearmatter and heavy-ion collision experiments, such as $\rho$ mass scaling versus broadening from dilepton spectra and chiral restoration signals in the $\sigma$ channel. We also elaborate on the molecular nature of $\pi \pi$ resonances.
\end{abstract}

PACS 11.10.Wx $\cdot$ 12.39.Fe $\cdot 21.65 .+f \cdot 25.75 .-\mathrm{q}$

\section{Introduction}

The lightest meson resonances, the $\rho(770)$ and the $f_{0}(600)$ or $\sigma$, play a crucial role in different phenomena pertaining to the hot and/or dense medium created both in relativistic heavy-ion collisions and in nuclear-matter experiments. The modifications of the spectral function of the $\rho$ resonance in medium are crucial to understand correctly the dilepton yield emerged from heavy-ion collisions $[1,2]$. The two main theoretical scenarios currently proposed in the literature can be classified into resonance mass shifting and broadening, according to the resulting dominant effect. Mass-shifting models are inspired by the Brown-Rho (BR) scaling hypothesis [3], which predicted that vector-meson

a e-mail: gomez@fis.ucm.es masses should scale with the quark condensate and therefore the main spectral modification of the resonance would be dictated by chiral-symmetry restoration. This scenario is supported also by the so-called hidden local-symmetry approach [4]. The broadening-dominated scenario is supported by various theoretical analyses [5-9] including recent unitarized chiral approaches [10-12]. The most recent experimental dimuon data from the NA60 Collaboration [2] clearly favor a broadening situation with negligible mass shift, whereas the earlier CERES results [1] were reasonably explained by both descriptions. It is worth to also mention the results of the STAR Collaboration at RHIC [13], which have reported a sizable mass reduction by medium effects measured in $\rho^{0} \rightarrow \pi^{+} \pi^{-}$instead of dileptons. The modifications of the $\rho$ properties have been also measured in cold nuclear-matter experiments. The E325-KEK collaboration [14] have reported a measurable shift in the masses of vector mesons compatible with theoretical predictions based on Brown-Rho scaling [15] and QCD sum rules [16]. On the other hand, the JLab-CLAS experiment [17] has obtained results compatible with vanishing mass shift, as predicted by most of the in-medium hadronic many-body analyses where broadening is the dominant effect $[5,7,10,18]$.

The possible modification of the $f_{0}(600) / \sigma$ in hot and dense matter is interesting, because this is a state with the same quantum numbers as the vacuum and therefore it might be sensitive to chiral-symmetry restoration. In this sense, an early proposal [19] suggested that the $\sigma$ could induce a measurable threshold enhancement of the $\pi \pi$ cross section, which would be interpreted as a precursor of chiralsymmetry restoration. The argument was that the mass of the $\sigma$ state should decrease by medium effects, since it is proportional to its vacuum expectation value in the chiral limit. Such a decrease would eventually shrink the available two-pion phase space when the $\sigma$ mass reaches the two-pion threshold, producing a bump in the imaginary part of the scattering amplitude, due to the proximity of the pole to the real axis. It is important to remark that in this original argument, it is implicitly assumed that i) the $\sigma$ is dominated by 
its $\bar{q} q$ component so that its expectation value behaves like the quark condensate and ii) that the $\sigma$ is narrow enough so that its width (imaginary part of the pole) vanishes when its mass (real part) approaches the threshold. None of these assumptions seem to be supported by the physical (vacuum) $f_{0}(600)$ state quoted by the Particle Data Group [20], which is a very broad state measured in $\pi \pi$ scattering. Although it is commonly accepted that this state is a member of the scalar nonet, its $\bar{q} q$ nature has been criticized on the basis of lattice [21] and large- $N_{c}$ [22] analyses. The physical state is likely to have important non- $\bar{q} q$ compound such as tetraquark, glueball or meson-meson, commonly referred to as a "molecular" state. Nevertheless, threshold enhancement is indeed observed in nuclear-matter experiments, both in $\pi A \rightarrow \pi \pi A^{\prime}[23,24]$ and in $\gamma A \rightarrow \pi \pi A^{\prime}$ [25] reactions. Although the size of the effect is still under debate, a clear signal is seen in the scalar channel for increasing nuclear density as compared with the vector channel and this is in fair agreement with most theoretical analyses at finite nuclear density [26-31]. In contrast, finite-temperature analyses show that this state remains broad even near the chiral phase transition despite the proximity of the pole to the twopion threshold $[12,30,32]$, which in practice does not produce any sizable enhancement in the scattering amplitude or cross section. A striking possibility to be explored is that by increasing further the medium strength, the $\sigma$ could become a $\pi \pi$ bound state, as suggested earlier in [33] and confirmed recently in $[12,30,32]$.

In the present work, we will investigate further about these issues, within the context of unitarized chiral approaches. The main goal is to establish to what extent chiral symmetry dictates the in-medium properties of the light meson resonances. The most general framework to account for all the interactions compatible with chiral symmetry is the effective chiral Lagrangian approach. The most prominent example is chiral perturbation theory (ChPT) for the meson sector [34, 35], but it can equally well be applied to the meson-baryon one [36]. Since these effective theories are built basically as expansions in derivatives or energies, they cannot account for resonances, since the chiral expansion violates the unitarity bounds. This has traditionally been solved by introducing unitarization methods, giving rise to the so-called chiral-unitary approaches, which have proved to be very successful in vacuum to describe meson-meson and meson-baryon interactions and generate dynamically low-lying resonances [39-45]. Furthermore, as commented above, the unitarization program has been extended to account for finite-temperature and density effects [10-12, 29, 31, 37, 38].

Here we will analyze some of our recent results for the $\rho$ and $\sigma$ mesons obtained within the unitarized chiral framework, paying special attention to their nature and their role in chiral-symmetry restoration and studying some aspects not considered before like a new analysis of the combined effects of temperature and nuclear density for the $\sigma$ meson based on a Lippmann-Schwinger (or Bethe-Salpeter) equation approach accounting for many-body pion dynamics versus a simplified $f_{\pi}$-scaling scenario, a direct comparison with nuclear-matter experiments of our results for the $\rho$ mass linear density dependence and the interpretation of the results obtained for the behavior of the resonances near threshold in terms of a "molecular" classification of those states.

This paper is organized as follows: in Sect. 2 we shall present the formalism and results within the framework of the inverse amplitude method (IAM) at finite temperature. In Sect. 3 we shall introduce nuclear-density effects only by rescaling properly the decay constant of the pion at $T=0$ within the IAM. In that section, we shall provide in particular an interpretation of our results in terms of "molecular" classification and a numerical comparison with experimental results from dilepton decays in resonance production in finite nuclei. Finally, in Sect. 4 we present a new calculation for the $\sigma$ channel, which includes temperature and nucleardensity many-body effects in order to compare our different approaches.

\section{Finite-temperature resonances with the inverse amplitude method}

One of the simplest and more powerful unitarization methods for chiral theories is the so-called inverse-amplitude method (IAM) [39-41]. Its name comes from the simple observation that unitarity implies that the inverse of a given partial-wave amplitude $t^{I J}$ in $\pi \pi \rightarrow \pi \pi$ scattering should satisfy

$$
\begin{aligned}
S^{\dagger} S=1 & \Rightarrow \operatorname{Im} t^{I J}(s)=\sigma_{0}(s)\left|t^{I J}(s)\right|^{2} \\
& \Rightarrow \operatorname{Im} \frac{1}{t^{I J}(s)}=-\sigma_{0}(s)
\end{aligned}
$$

for $s>4 m_{\pi}^{2}$, where $s$ is the center of mass energy squared and $\sigma_{0}(s)=\sqrt{1-4 m_{\pi}^{2} / s}$ is the two-pion phase space.

Consider now the ChPT expansion of partial-wave amplitudes:

$t^{I J}(s)=t_{2}^{I J}(s)+t_{4}^{I J}(s)+\mathcal{O}\left(p^{6}\right)$.

Here, $p$ denotes generically a meson momentum, mass or temperature ( $p$ is to be compared with the characteristic chiral scale $\Lambda_{\chi} \sim 1 \mathrm{GeV}$, whereas $T$ is meant to be below $\left.T_{\mathrm{c}} \simeq 200 \mathrm{MeV}\right)$ and $t_{k}$ is the $\mathcal{O}\left(p^{k}\right)$ contribution. Recall that, according to standard ChPT power counting [34, 35], $t_{2}$ accounts for tree-level diagrams from the lowest-order Lagrangian $\mathcal{L}_{2}$. Up to that order, only the pion decay constant 
$f_{\pi}$ and the pion mass $m_{\pi}$ enter the result. The order $t_{4}$ includes the one-loop diagrams from $\mathcal{L}_{2}$ plus the tree-level $\mathcal{L}_{4}$ terms needed for renormalization. The $\mathcal{L}_{4}$ low-energy constants entering the pion-scattering amplitude, when it is expressed in terms of the physical $m_{\pi}$ and $f_{\pi}$ are denoted $\bar{l}_{1}-\bar{l}_{4}$ in the convention of [35].

The chiral expansion (2.2) satisfies only a perturbative version of the unitarity relation (2.1), namely

$\operatorname{Im} t_{4}^{I J}(s)=\sigma_{0}(s)\left|t_{2}^{I J}(s)\right|^{2}$

and so on for higher orders, which eventually means that chiral expansions are not compatible with the bounds on partial waves implied by unitarity. In other words, they grow arbitrarily with energy. Unitarization methods allow one to construct chiral amplitudes that are exactly unitary. In particular, the IAM amplitudes are built by demanding i) exact unitarity and ii) that at low energies they match the ChPT series to a given order. These conditions lead to the IAM result, which is formally justified by the use of dispersion relations [39-41]. There is, however, a further, more technical requirement, which is that the IAM partial-wave amplitudes should vanish at the same values of the energy and with the same power as the perturbative amplitudes. These values are the so-called Adler zeros and lie below threshold. Since a zero of the amplitude is a pole of its inverse, this affects the analytic structure of $1 / t$. A detailed discussion can be found in [46] where the proper correction to the IAM is derived using dispersion relations and it is shown that these additional terms produce a negligible effect in the physical region. However, as discussed in [12], taking into account this correction is important when dealing with medium effects that can drive the poles to the real axis, as is the case here, since otherwise there would be spurious poles both in the first and second Riemann sheets below threshold.

The IAM can be extended at finite temperature by including the thermal corrections to the scattering amplitude, which have been calculated in [47] to one loop in ChPT. Since temperature enters only in the loops, $t_{2}$ is $T$ independent. For $t_{4}(s ; T)$ one gets a perturbative unitarity relation exactly like (2.2) but with the phase space replaced by

$\sigma_{\mathrm{T}}(s)=\sigma_{0}(s)\left[1+2 n_{\mathrm{B}}(\sqrt{s} / 2)\right]$,

with $n_{\mathrm{B}}(x)=[\exp (x / T)-1]^{-1}$ the Bose-Einstein distribution function.

The function $\sigma_{\mathrm{T}}(s)$ is the thermal phase space, which is increased with respect to the $T=0$ one by the difference $\left[1+n_{\mathrm{B}}\left(E_{1}\right)\right]\left[1+n_{\mathrm{B}}\left(E_{2}\right)\right]-n_{\mathrm{B}}\left(E_{1}\right) n_{\mathrm{B}}\left(E_{2}\right)=1+n_{\mathrm{B}}\left(E_{1}\right)+$ $n_{\mathrm{B}}\left(E_{2}\right)$, where $E_{1,2}$ are the energies of the two colliding pions, corresponding to the difference between enhancement due to the increase of two-pion outgoing states and absorption due to collisions of the incoming pions with the thermal bath ones. In the center-of-mass frame, in which partial waves are defined, $E_{1}=E_{2}=\sqrt{s} / 2$, and the thermal phase space reduces to (2.4). One can then use the same $T=0$ IAM requirements, replacing $\sigma_{0} \rightarrow \sigma_{\mathrm{T}}$ and the partial waves by the finite- $T$ ones, provided that only intermediate twopion states are relevant in the thermal bath, as expected in a dilute-gas regime at low and moderate temperatures. Finally, one arrives at the thermal IAM formula for a given partial wave:

$$
\begin{aligned}
t^{\mathrm{IAM}}= & \frac{t_{2}(s)^{2}}{t_{2}(s)-t_{4}(s ; T)+A(s ; T)}, \\
A(s ; T)= & t_{4}\left(s_{2} ; T\right) \\
& -\frac{\left(s_{2}-s_{A}\right)\left(s-s_{2}\right)}{s-s_{A}}\left[t_{2}^{\prime}\left(s_{2}\right)-t_{4}^{\prime}\left(s_{2} ; T\right)\right],
\end{aligned}
$$

where the $A$ function is the Adler zero contribution discussed above, $s_{\mathrm{A}}$ denoting the Adler zero ( $T$-dependent) expanded as $s_{\mathrm{A}}=s_{2}+s_{4}+\cdots$ with $s_{4}=-t_{4}\left(s_{2} ; T\right) / t_{2}^{\prime}\left(s_{2}\right)$.

Performing the conventional extension of the amplitude to the second Riemann sheet, one finds poles in the $I=$ $J=0$ and $I=J=1$ channels, which are identified as the $f_{0}(600)$ and the $\rho(770)$. We show in Fig. 2.1 the results for the pole position $s_{\text {pole }}=\left(M_{\mathrm{p}}-\mathrm{i} \Gamma_{\mathrm{p}} / 2\right)^{2}$ for different temperatures. The $\bar{l}_{i}$ values we have used are $\bar{l}_{1}=-0.3, \bar{l}_{2}=5.6$, $\bar{l}_{3}=3.4$ and $\bar{l}_{4}=4.3$, which give for the mass and width of the $\rho(770)$ at $T=0 M_{\mathrm{p}} \simeq 756 \mathrm{MeV}$ and $\Gamma_{\mathrm{p}} \simeq 151 \mathrm{MeV}$. For the $f_{0}(600) / \sigma$ at $T=0$ we find $M_{\mathrm{p}} \simeq 441 \mathrm{MeV}$ and $\Gamma_{\mathrm{p}} \simeq 464 \mathrm{MeV}$.

The general features we observe are that the thermal $\rho$ pole shows a predominant and increasing broadening behavior, while for the $\sigma$ an important mass decrease takes place, presumably due to chiral restoration, while the width increases for low temperatures but decreases for temperatures of $T \simeq 100 \mathrm{MeV}$ and beyond. In the rest of this section, we shall discuss in more detail these different behaviors in connection with the phenomenological issues commented on in the introduction.

2.1 The thermal $\rho$ meson: broadening versus mass scaling in $\pi \pi$ scattering and dilepton probes

The $\rho$ pole obtained in our IAM thermal approach undergoes a significant broadening at finite temperature. The main source of thermal broadening is the Bose-Einstein increase of phase space given in (2.4). However, it is not the only one. In fact, using the Breit-Wigner $\left(\Gamma_{\mathrm{p}} \ll M_{\mathrm{p}}\right)$ parametrization for the $\rho$ exchange in $\pi \pi \rightarrow \rho \rightarrow \pi \pi$, one gets [11]

$$
\frac{\Gamma_{\mathrm{T}}}{\Gamma_{0}}=\left[1+2 n_{\mathrm{B}}\left(M_{\mathrm{T}} / 2\right)\right] \frac{g_{\mathrm{T}}^{2} M_{\mathrm{T}}}{g_{0}^{2} M_{0}},
$$

where $g$ is the effective $\rho \pi \pi$ vertex.

In Fig. 2.2 (left) we show in detail the dependence of the mass, width and effective vertex, and it is clearly seen 

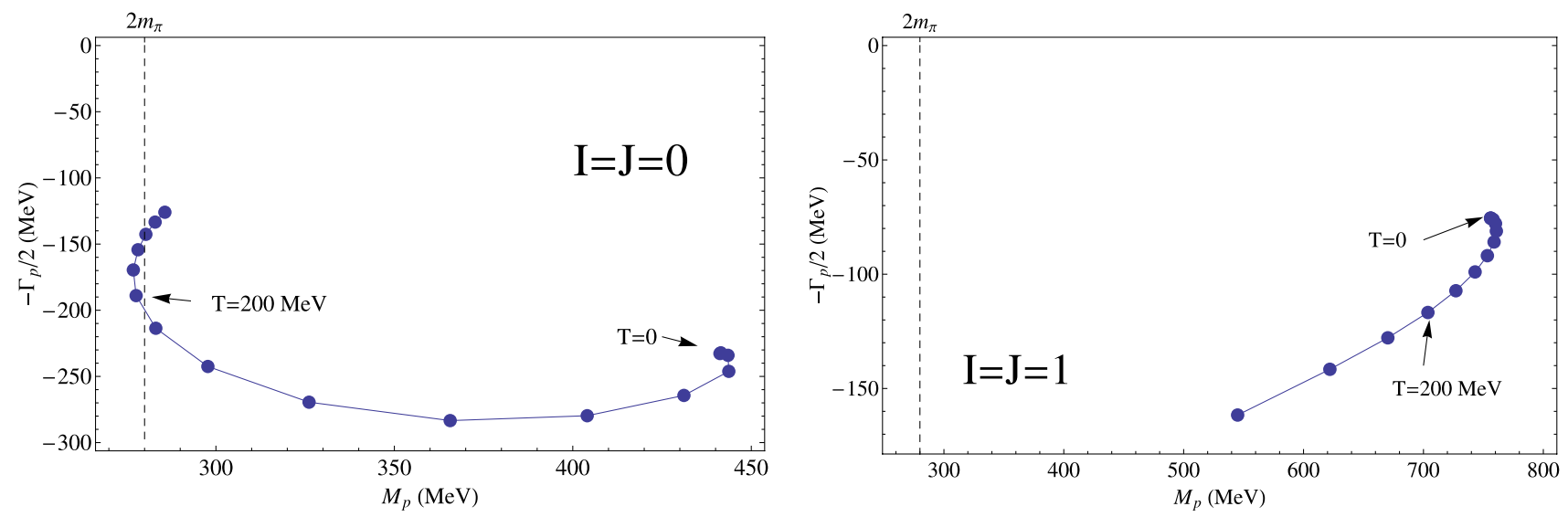

Fig. 2.1 Temperature dependence of the $f_{0}(600)$ and $\rho$ complex poles unitarized by the IAM. The points are obtained by varying the temperature in $20 \mathrm{MeV}$ intervals
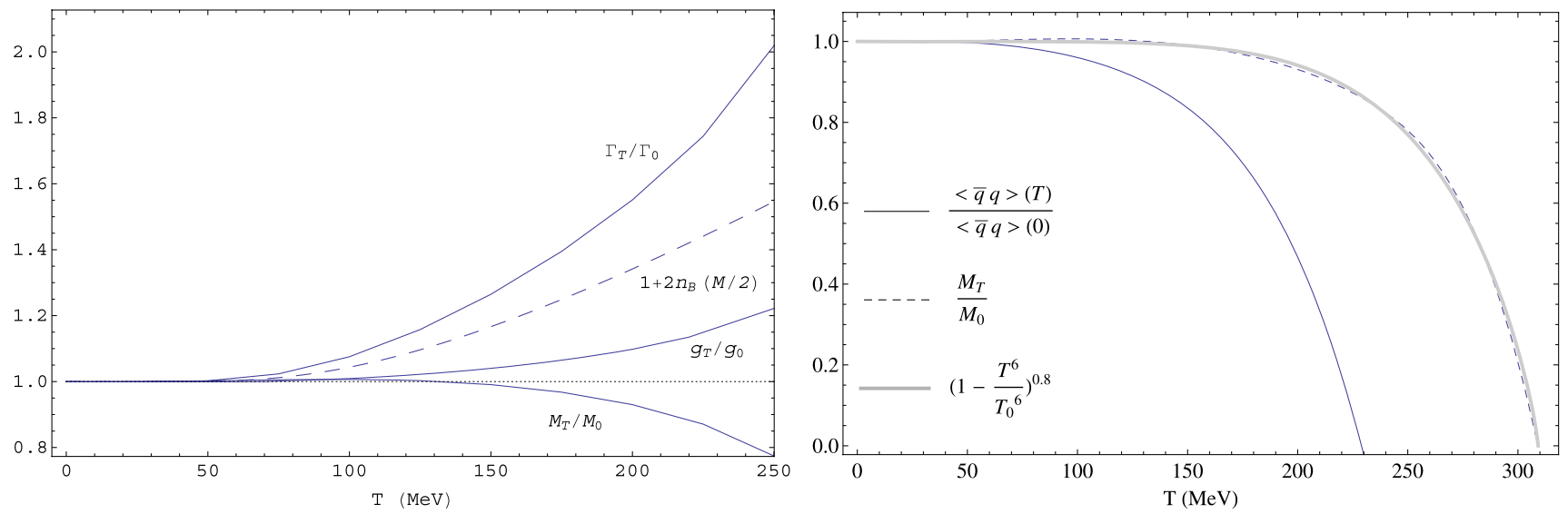

Fig. 2.2 Thermal width and mass of the $\rho$ as extracted from its IAM pole. In the left panel we also show the $T$-dependence of the effective $\rho \pi \pi$ vertex. In the right panel we compare the mass with the quark condensate. The mass vanishes at $T_{0} \simeq 310 \mathrm{MeV}$

that the width increases roughly as the phase space up to $T \simeq 100 \mathrm{MeV}$, and then further broadening arises due to the increasing effective $\rho \pi \pi$ vertex. The broadening we have obtained is also present in the electromagnetic pion form factor [48], which enters directly into the dilepton yield (for back-to-back dileptons in our case) arising from $\pi^{+} \pi^{-} \rightarrow l^{+} l^{-}$. Our result is therefore compatible with the broadening scenario observed clearly in NA60 [2] (see the discussion in the introduction).

The mass of the pole barely changes with temperature in our approach. This seems to be in contradiction with the dropping-mass scenario obtained in scaling models, which predict that the mass should scale roughly with the quark condensate $M_{\mathrm{T}} / M_{0} \simeq\langle\bar{q} q\rangle_{\mathrm{T}} /\langle\bar{q} q\rangle_{0}$ [3]. However, it is worth mentioning that more recent analyses based on the same scaling hypothesis $[4,49]$ suggest that the mass dropping might be really effective only very close to the transition temperature, which in practice would mean that those predictions are not strictly incompatible with the dilepton data.

In order to clarify our results on this matter, we have plotted in Fig. 2.2 (right) the pole mass and the quark condensate calculated from a virial expansion [50] using the $\mathcal{O}\left(p^{4}\right)$ pion-scattering amplitudes, which gives a critical temperature $T_{\mathrm{c}} \simeq 230 \mathrm{MeV}$. The results are extrapolated up to the temperature $T_{0}$, where the mass vanishes, although our ChPT-based approach is not meant to be valid there. We see that the mass drops rather abruptly, with a $T^{6}$ power, while, as said before, it remains almost constant for $T$ below the chiral transition. We do not see a scaling pattern when compared to the condensate and, besides, $T_{0} \simeq 310 \mathrm{MeV}$ lies far from the critical value where the condensate vanishes. On the other hand, in BR-like scaling models, the effective vertex decreases [4] and, as commented before, there is no significant broadening. In conclusion, although we obtain a dropping mass qualitatively compatible with BR-like models, our dominant broadening effect, the increase of the ef- 

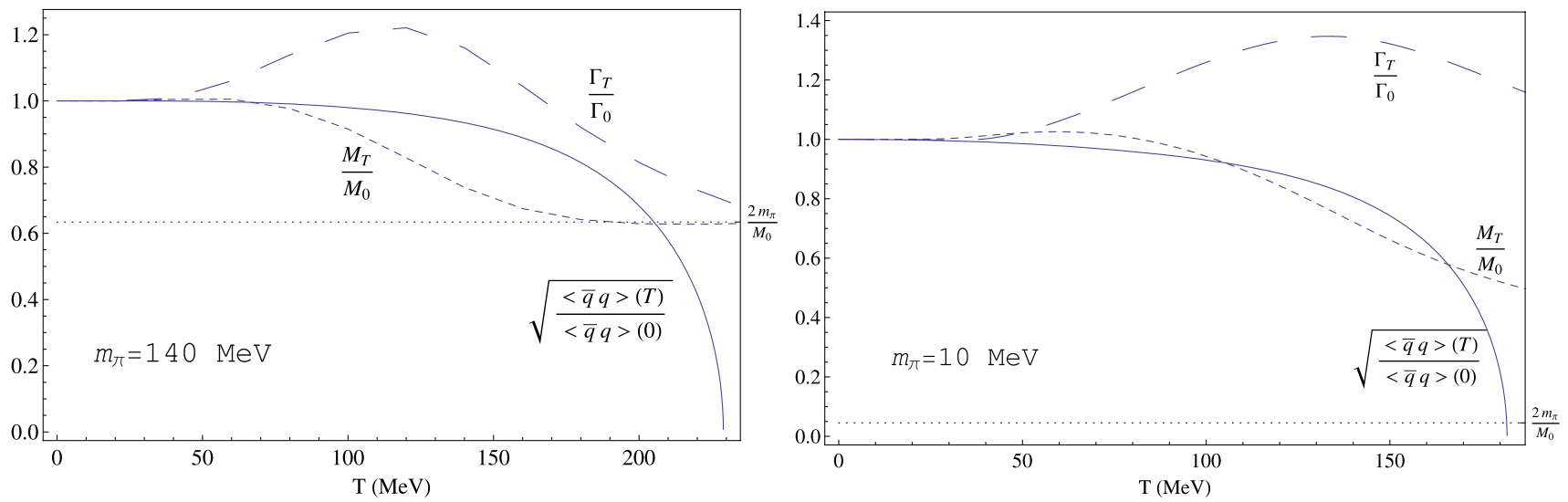

Fig. 2.3 Comparison between the $\sigma / f_{0}(600)$ mass (extracted from the IAM pole) at finite temperature and the root of the condensate, for the physical pion mass and near the chiral limit

fective vertex and the departure from the condensate are in conflict (at finite temperature) with that scenario.

\subsection{The thermal $f_{0}(600) / \sigma$ meson: threshold behavior and $\bar{q} q$ nature}

As mentioned in the introduction, the interest in the $\sigma$ pole concerns mainly its role as a precursor of the phase transition. The temperature dependence we obtain in Fig. 2.1 shows that a decreasing mass is a prominent feature in this channel, thus signaling chiral restoration. In fact, this is the dominant effect here over the phase-space increase for $T \simeq$ $100 \mathrm{MeV}$ and beyond. However, and this is crucial as far as observable effects are concerned, the pole remains broad even when its real part has reached the two-pion threshold. The reason behind this is that for a broad state $\left(\Gamma_{\mathrm{p}} \sim M_{\mathrm{p}}\right)$ the usual result $\Gamma \propto \sigma\left(M^{2}\right) \theta\left(M^{2}-4 m_{\pi}^{2}\right)$, which forbids the pole to be at threshold with a nonzero width, should be replaced by $\Gamma \sim \int \sigma(s) \theta\left(s-4 m_{\pi}^{2}\right) \rho(s)$ [12], where $\rho(s)$ is the resonance spectral function, different in general from the narrow $\delta$-function $\rho(s)=2 \pi \delta\left(s-M^{2}\right)$. Therefore, the width can remain sizable near threshold. In fact, a simple model of thermal width based on this observation turns out to reproduce quite well the obtained IAM poles [12]. This behavior for the $\sigma$ pole implies no threshold enhancement at finite temperature as a precursor of the transition, since the pole is still far from the real axis. This is clearly observed when we plot the squared modulus of the $I=J=0$ partial wave in Fig. 3.3 (left). Threshold enhancement for a typical narrow resonance would mean for the amplitude $\operatorname{Im} t(s) \sim 2 m_{\pi} \theta\left(s-4 m_{\pi}^{2}\right) / \sqrt{s-4 m_{\pi}^{2}}$ for $s \lesssim 4 m_{\pi}^{2}$ [12]. As we shall see below, the situation changes dramatically when finite-density effects are included.

The role of the $\sigma$ pole as a precursor of the transition is strongly linked to its $\bar{q} q$ nature, as discussed in the introduction. In Fig. 2.3 we compare the mass of the pole with the root of the quark condensate, similarly as we did with the $\rho$ in the previous section. Recall that, in a $O(4)$ model, $M_{\sigma} \sim\langle\sigma\rangle \sim f_{\pi}$ and, on the other hand, $f_{\pi}^{2}=-m_{q}\langle\bar{q} q\rangle / m_{\pi}^{2}$ from the Gell-Mann-Oakes Renner (GOR) relation [51]. We also plot the results near the chiral limit, where the condensate vanishes at a lower temperature and explicit chiralsymmetry breaking effects are minimized. In any case, we do not see a scaling pattern, as expected from our result of a broad thermal state. Near the chiral limit, the pole mass does not even go to threshold near the critical temperature, since the width is notably increased due to the higher volume of available phase space. From our analysis of the thermal effects one can also conclude that the non- $\bar{q} q$ component of the $f_{0}(600)$ must be of crucial importance. As discussed in the introduction, the same conclusion has been reached in vacuum studies [21, 22]. The novelty here is the use of thermal arguments. We shall see in Sect. 3 that finite-density effects driving the resonance poles to the real axis below threshold also allow one to extract interesting conclusions about their $\bar{q} q$ nature.

\section{Finite-density chiral restoring effects at $T=0$ through $f_{\pi}$ scaling in the IAM}

The simplest way to incorporate nuclear-density effects at $T=0$ in the unitarized chiral approach is to encode them only in the variation of the pion decay constant, to linear order in density, as $[52,53]$ follows:

$$
\begin{aligned}
\frac{f_{\pi}^{2}(\rho)}{f_{\pi}^{2}(0)} & \simeq \frac{\langle\bar{q} q\rangle(\rho)}{\langle\bar{q} q\rangle(0)} \simeq\left(1-\frac{\sigma_{\pi N}}{m_{\pi}^{2} f_{\pi}^{2}(0)} \rho\right) \\
& \simeq\left(1-0.35 \frac{\rho}{\rho_{0}}\right)
\end{aligned}
$$


where $\rho$ is the nuclear density, $\sigma_{\pi N} \simeq 45 \mathrm{MeV}$ is the pionnucleon sigma term and $\rho_{0} \simeq 0.17 \mathrm{fm}^{-3}$ is the normal or saturation nuclear-matter density.

Therefore, by varying $f_{\pi}$ in our IAM approach, we mimic chiral restoring nuclear effects [12]. This approach clearly ignores standard many-body corrections, like the coupling of pions to particle-hole $(p h)$ and Delta-hole $(\Delta h)$ excitations, considered for instance in $[8,10,26,31]$. This approximation is meant to be more adequate for $\sigma$ than for $\rho$, since, as we have seen at finite temperature, chiral restoration tends to dominate the $\sigma$ pole behavior under medium effects. In fact, by changing only $f_{\pi}$, no medium broadening is produced, which is probably unrealistic for the $\rho$ case, as emphasized in many-body work $[5,7,10]$. This must be borne in mind when interpreting our results for the $\rho$ in terms of BR-scaling. In any case, in Sect. 4 we shall consider a different unitarization scheme, which allows us to introduce all the above mentioned nuclear many-body ef- fects and to compare with our simple " $f_{\pi}$ scaling" considered here.

The results obtained by varying $f_{\pi}$ in the IAM amplitudes are displayed in Figs. 3.1 and 3.2 for the pole trajectories in the complex plane and mass scaling, respectively, in the 00- and 11-channels. In Fig. 3.3 (right) we also show the effect on the scattering amplitude in the $\sigma$ channel. As $f_{\pi}$ decreases, the $\sigma$ pole becomes narrow enough so that chiral mass reduction brings it to the real axis, which produces threshold enhancement in the amplitude although at densities well above $\rho_{0}$. Regarding the mass dropping, it takes place now along with the condensate (see Fig. 3.2). In Fig. 3.4 we show $\left|t_{00}\right|^{2}$, considering simultaneously the temperature and finite density according to (3.1) for $f_{\pi}$. We observe that, for a given value of $f_{\pi}$ (small enough), the net effect of introducing temperature is to amplify threshold enhancement, as the amplitude is notably softened at higher energies, by thermal broadening, what makes the low-energy region relatively more important. Still, one has
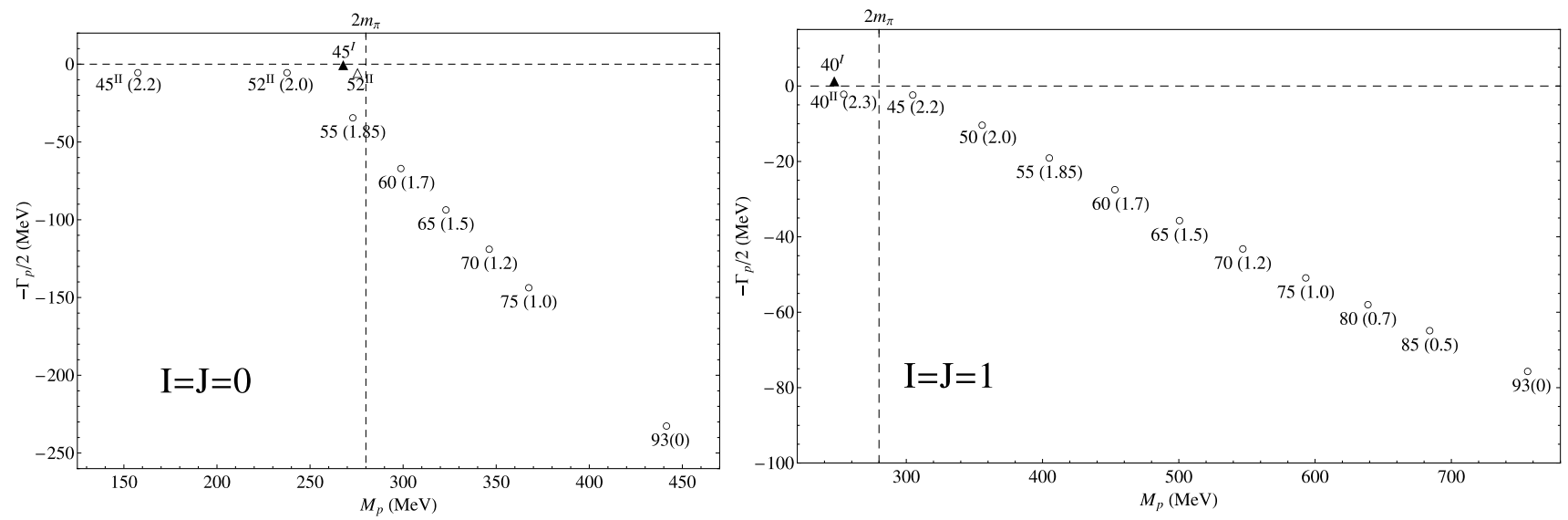

Fig. 3.1 IAM poles with varying $f_{\pi}$. The numbers attached to the pole positions indicate $f_{\pi}(\rho)\left(\rho / \rho_{0}\right.$ in brackets) in MeV according to (3.1). The circles and open triangles denote second-sheet poles, while black triangles refer to first-sheet ones
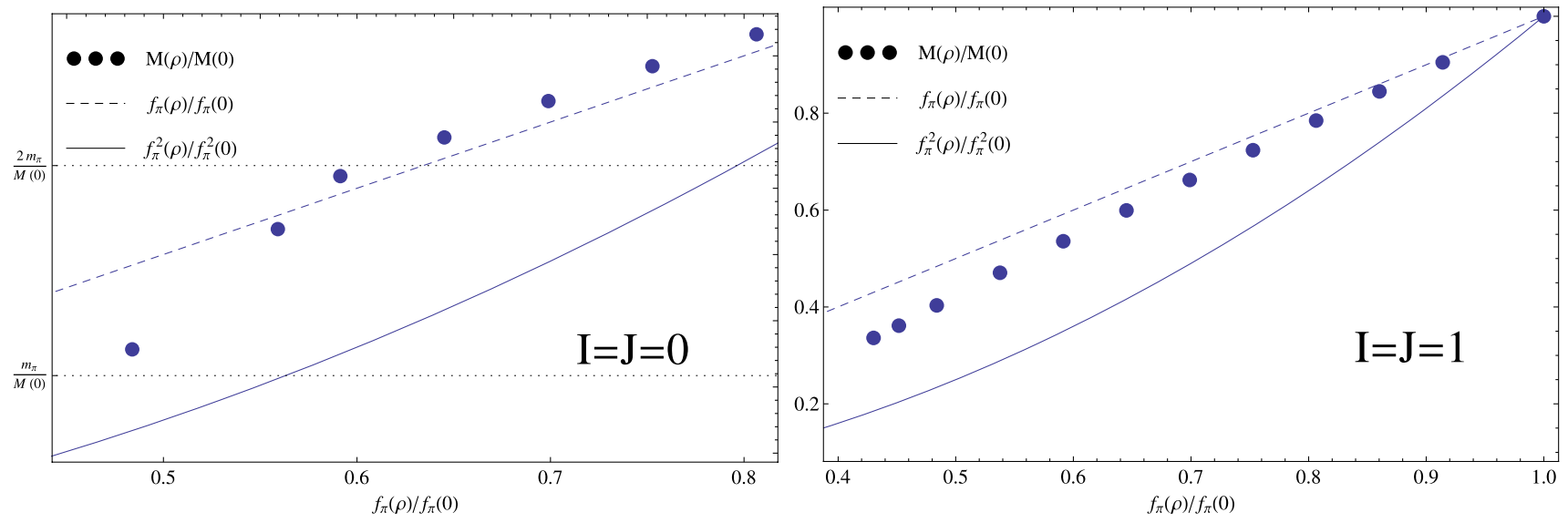

Fig. 3.2 Scaling of the mass, compared with $f_{\pi}^{2}(\rho) / f_{\pi}^{2}(0) \sim\langle\bar{q} q\rangle(\rho) /\langle\bar{q} q\rangle(0)$ and with $f_{\pi}(\rho) / f_{\pi}(0)$. In the $I=J=0$ case, the masses displayed correspond to the lowest masses of the second-sheet poles 

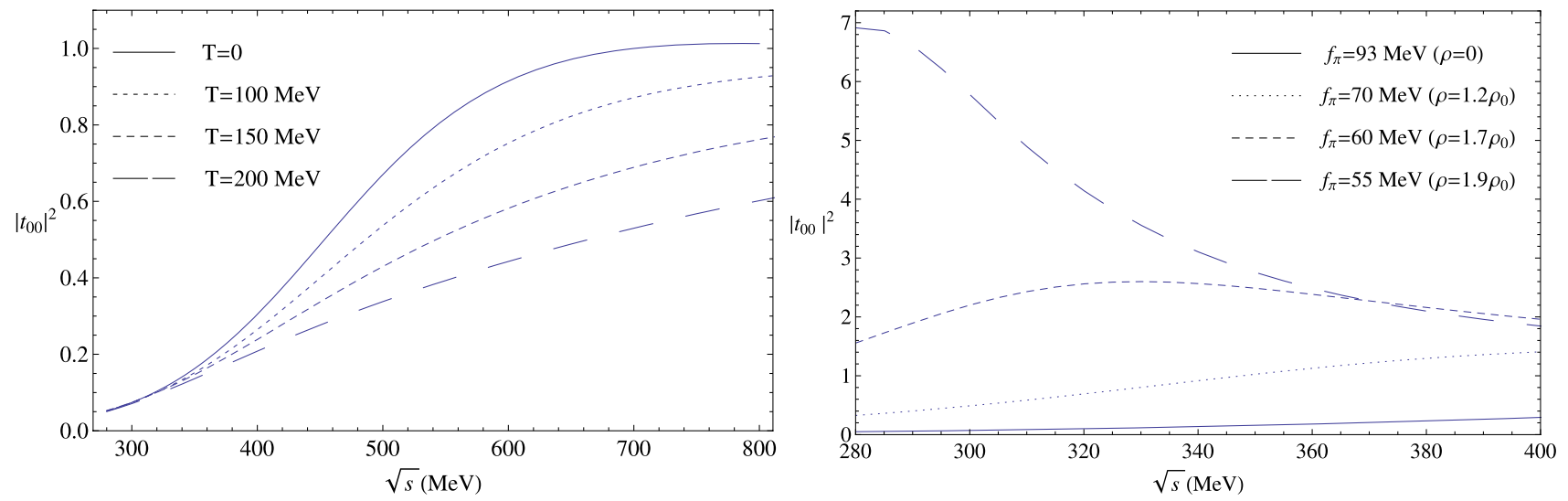

Fig. 3.3 Squared modulus of the $I=J=0$ partial wave at finite temperature (left) and at $T=0$ and varying $f_{\pi}$ (right) to simulate chiral restoration at finite density
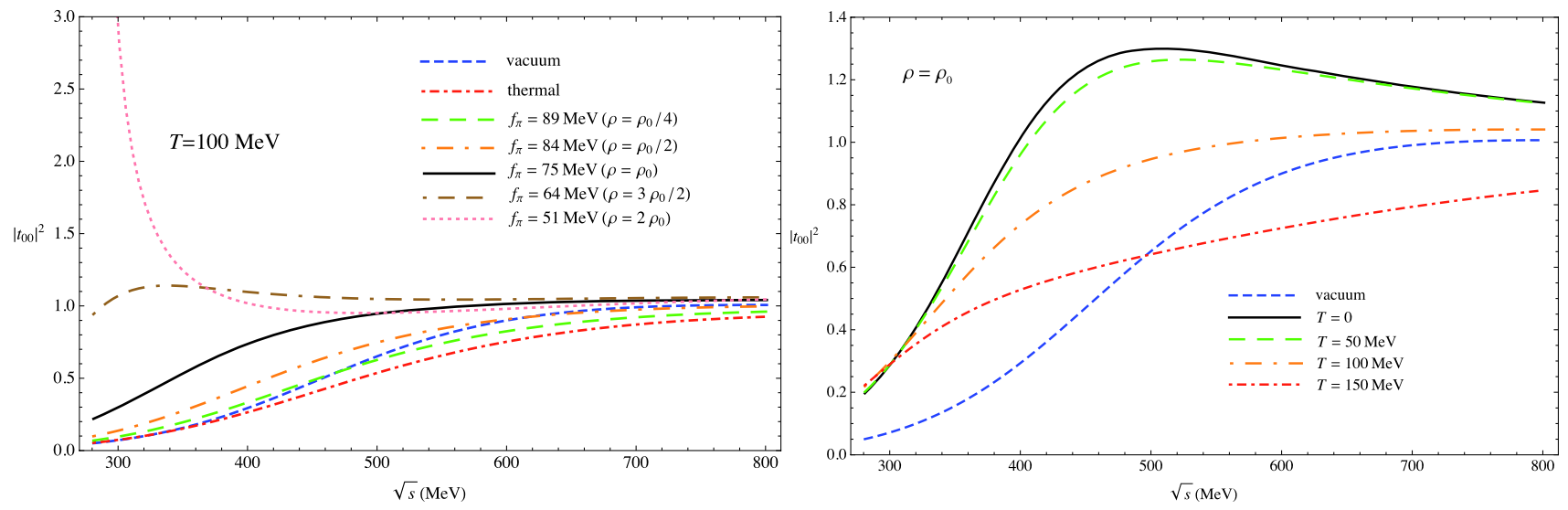

Fig. 3.4 Squared modulus of the $I=J=0$ partial wave at $T=100 \mathrm{MeV}$ for several densities (left), and at nuclear-matter density $\left(f_{\pi}=75 \mathrm{MeV}\right)$ for several temperatures (right)

to keep in mind that (a) this effect is driven by the proximity of the $\sigma$ pole to the real axis when decreasing $f_{\pi}$, (b) thermal effects alone do not generate threshold enhancement in the amplitude as discussed above, and (c) the $\pi \pi$ phase space is not open below $2 m_{\pi}$ even at finite temperature. In Sect. 4 we shall compare these results for the 00-channel with an implementation of nuclear-density effects in a dynamical many-body calculation.

As for the $\rho$ channel, the BR-like scaling pattern is now closely followed. In fact, our $\rho$-meson pole moves gradually from the $\sqrt{\langle\bar{q} q\rangle}$ curve to the $\langle\bar{q} q\rangle$ one, as obtained in [54]. However, one must be careful about this conclusion, since we are disregarding medium-related broadening, which might change the scaling picture observed here. Strictly speaking, our results indicate that if the relevant density effects amount only to a scaling of $f_{\pi}$, then one gets scaling in the pole mass, which is quite consistent with the BR idea.

There is an additional interesting feature of our results: when the density is increased further, $\pi \pi$ bound states (first- sheet poles) appear just below threshold in both channels. As we shall see in Sect. 3.2, this result allows for an interpretation in terms of a "molecular" classification of resonances, which is completely different in both channels. In fact, we note that in the $\sigma$ channel the bound state is preceded by a doubling of poles in the second sheet. This will be further discussed in Sect. 3.2. The appearance of pole doubling and bound states has been also analyzed in other work [30, 32, 33].

\subsection{Comparison with nuclear-matter experiments}

As discussed above, apart from heavy-ion collisions, there are several dedicated experiments on production reactions in nuclei where our analysis can be useful, namely, those related to threshold enhancement in $\pi \pi$ production and the modification of dilepton spectra from $\rho$ decays.

The threshold enhancement observed in [23-25] in the $I=J=0$ channel is qualitatively reproduced in our " $f_{\pi}$ 
scaling" finite-density approach. Comparing with our finite$T$ analysis in the previous sections, our main conclusion is that, due to the broad nature of the $\sigma$ at finite $T$, threshold enhancement is not visible at finite $T$ and it can only take place if chiral restoring finite-density effects are strong enough, compared for instance with medium effects we have neglected which could also increase the $\sigma$ width, preventing the pole from approaching the real axis, as in the finite- $T$ case, and erasing the threshold effect. This does not seem to be the case when many-body interactions are properly accounted for [26, 29], which gives support to this simple approximation. In fact, the observed effect is in reasonable quantitative agreement with previous theoretical works $[27,28,30]$, and it is not very strong for densities $\rho \lesssim \rho_{0}$ (the range available experimentally) in line with the experimental results in [24].

As for dileptons in nuclear matter, a parameter directly measurable in those experiments is the coefficient of the linear-density term for the mass of the $\rho(770)$ meson:

$$
\frac{M(\rho)}{M(0)}=1-\alpha \frac{\rho}{\rho_{0}}
$$

The experimental values available so far are $\alpha=0.092 \pm$ 0.002 obtained by the E325-KEK collaboration [14] and $\alpha=0.02 \pm 0.02$ measured by the JLab-CLAS experiment [17]. We have performed a linear fit of our pole results in the $I=J=1$ channel for $f_{\pi}=93,85,80,75 \mathrm{MeV}$, i.e., up to $\rho \lesssim \rho_{0}$, in order to be closest to the experimental situation. Our fit gives $\alpha=0.2$, which is closer to the result in [14] than to the one in [17], although a bit above the experimental value. Our result is also in agreement with Brown-Rho scaling [15] and QCD sum rules [16], which predict $\alpha \simeq 0.1-0.2$. It is important to remark again that so far we ignore all medium-broadening effects, which, unlike the case of the $\sigma$, may be crucial in this case, as emphasized in many-body treatments [5, 7, 10, 18]. In fact, in [55] it was realized that QCD sum rules themselves do not provide a unique constraint for the in-medium mass and width variation, unless additional model assumptions are made. In particular, it is shown that if one assumes that the width is not increased then automatically the mass drops. This is the scenario we have recovered in our present approach, which, as commented several times above, does not mean that this is the physically relevant case.

\section{2 "Molecular" classification of resonances}

One of the objectives of this work is to analyze the $\bar{q} q$ structure of light meson resonances from a thermal and finitedensity viewpoint. We have already discussed in Sect. 2.2 that our results for the thermal $\sigma$ are not consistent with its pure $\bar{q} q$ nature.
The situation changes qualitatively with the $T=0$ finitedensity dependence obtained in Sect. 3. We see in Fig. 3.2 that the $\sigma$ pole follows quite well the same pattern of a $O(4)$ $\sigma$-like $\bar{q} q$ state. One may then wonder about the implication of this for the $\bar{q} q$ nature of the in-medium $\sigma$. We want to point out here that actually one can gain very useful information about that by looking at the behavior of the poles near threshold. Our argument is supported by the classification of resonances lying near threshold given in $[56,57]$ and based on the effective-range approximation. In those works it is stated that although generally it is difficult to extract properties about the "internal" nature of resonances from its decay products (scattering poles), when the pole lies near threshold, a general rule can be applied: a "potential" or "molecular" resonance shows up as a single pole near threshold, while for a $\bar{q} q$-like state two poles near threshold appear very close to another in different Riemann sheets. This classification was originally applied to states like the $f_{0}(980)$, which lies very close to the $\bar{K} K$ threshold.

Our claim here is that this classification argument can equally be applied to light resonances if medium effects drive the poles to the real axis. This is exactly the situation when we include density effects only through (3.1), as is clear from the results in Fig. 3.1, although bearing in mind that the effective values of $f_{\pi}$ for which the poles reaches the real axis are too small to trust entirely our ChPT-based approach. We see that the $\sigma$ pole follows a clear "molecular" pattern, since the pole that remains close to threshold and eventually becomes a bound state is well separated from the second-sheet pole that lies below threshold. We interpret this as the coexistence of two states for high enough densities: a $\pi \pi$ "molecule" and a virtual state which behaves as a "chiral partner" of the pion, in the sense that it tends to become degenerate in mass with it (albeit with different quantum numbers) following the order parameter. Note that the pole we represent in Fig. 3.2 is precisely this virtual state. This picture is in contrast with what we observe for the $\rho$ channel, where two nearby poles remain below threshold, one of them moving to the first sheet and becoming a bound state. This is clearly a $\bar{q} q$, non-molecular scenario, according to the previously discussed classification. We remark that a similar picture for the real axis poles has been obtained in [30], where the density dependence is also parametrized in $f_{\pi}$ and in vacuum by increasing the quark mass in order to compare with lattice results [58].

\section{Finite temperature and density in a many-body unitarized approach}

In this section we extend our previous results in the $I=J=$ 0 channel by incorporating finite nuclear-density and temperature effects in a many-body description of pion dynamics. We follow the line of Refs. [26, 31] where the effective 
$\pi \pi$ scattering amplitude in cold nuclear matter was evaluated in a chiral unitary framework. For technical reasons which we discuss below, it is convenient to use a different unitarization scheme for the $t$-matrix, which however provides similar results in vacuum to those from the IAM, i.e., the light meson-meson resonances are dynamically generated in the scalar channel and many scattering observables are described in good agreement with experiment [59-61].

The idea is to solve the coupled-channel Bethe-Salpeter (BS) equation for the partial-wave scattering amplitude (in matrix notation),

$T=V+\overline{V G T}$

where the potential (kernel) $V$ of the equation is provided by the lowest-order tree-level amplitude from the chiral Lagrangian. In (4.1),

$$
\begin{aligned}
G_{i}(P)= & \mathrm{i} \int \frac{\mathrm{d}^{4} q}{(2 \pi)^{4}} \frac{1}{\left(P^{0}-q^{0}\right)^{2}-\boldsymbol{q}^{2}-m_{i}^{2}+\mathrm{i} \varepsilon} \\
& \times \frac{1}{\left(q^{0}\right)^{2}-\boldsymbol{q}^{2}-m_{i}^{2}+\mathrm{i} \varepsilon}
\end{aligned}
$$

stands for the intermediate two-particle meson-meson Green's function of channel $i$ ( $G$ is diagonal), where $P=$ $\left(P^{0}, \mathbf{0}\right)$ is the total external momentum in the center of mass frame of the two pions (rest frame with respect to the nuclear medium), with $s=\left(P^{0}\right)^{2}$. In principle, both $V$ and $T$ enter off-shell under the momentum integration $(\overline{V G T}$ term) of the meson-meson loop. However, as was shown in Ref. [59], the (divergent) off-shell contributions of $V$ and $T$ in the $s$-wave interaction can be reabsorbed in a renormalization of the bare coupling constants and masses order by order. Therefore, both $V$ and $T$ can be factorized onshell, leaving the four-momentum integration only in the two-particle meson-meson propagator; cf. (4.2). An alternative justification for solving the Bethe-Salpeter equation with on-shell amplitudes can be found in the framework of the $N / D$ method, applied for meson-meson [62] and meson-baryon [63] interactions. We are thus left with a set of linear algebraic equations with trivial solution,

$T=[1-V G]^{-1} V$.

Since we are interested in the $\sigma$ meson energy region (and particularly close to the two-pion threshold), for simplicity we shall work in a single-channel approach (the coupled $\bar{K} K$ channel lies far above in energy and has little effect at low energies). The conditions under which this unitarization procedure is equivalent to the IAM were discussed in $[60,61]$. The main difference of the present approach as compared to the full IAM amplitude lies in the $\mathcal{O}\left(p^{4}\right)$ contribution (which here comes from the $s$-channel mesonmeson loop; and no tadpole or $\mathcal{L}_{4}$ tree-level terms are included) and the absence of $t$ - and $u$-channel diagrams (or,

in other words, the left-hand analytical cut is ignored). Still, the present scheme dynamically generates the $\sigma$ pole with similar properties as in the IAM and the experimental phase shifts in $\pi \pi$ scattering are well reproduced [59-61]. Also note that (2.1), (2.4) are equally satisfied in this approach. We show in Fig. 4.1 a diagrammatic representation of the series of $s$-channel diagrams which is summed in the BS equation.

The calculation of the thermal amplitude proceeds by first reanalyzing the on-shell factorization of $V$ and $T$ in the BS equation. At finite temperature, the divergent contribution from off-shell terms in the one-loop amplitude has the same structure as in vacuum, but a finite, temperature-dependent part survives, which cannot be cast as a (vacuum) renormalization of $f_{\pi}$ or $m_{\pi}$. This contribution can be accounted for as a temperature correction to the $\mathcal{O}\left(p^{2}\right)$ kernel,

$\delta V_{\mathrm{off}}^{T}(s)=\frac{4}{3 f_{\pi}^{2}}\left(V_{\mathrm{on}}(s)+\frac{s}{3 f_{\pi}^{2}}\right) I_{0}^{T}$,

with $I_{0}^{T}=\left(2 \pi^{2}\right)^{-1} \int_{0}^{\infty} \mathrm{d} q q^{2} n_{\mathrm{B}}\left(\omega_{q}\right) / \omega_{q}, V_{\text {on }}(s)=-(s-$ $\left.m_{\pi}^{2} / 2\right) / f_{\pi}^{2}$ and $\omega_{q}^{2}=\boldsymbol{q}^{2}+m_{\pi}^{2}$ (we follow in this section the normalization of partial waves given in $[59,60]$, which differs from the one in Sect. 2 in a factor $16 \pi$ ). In order to keep as close as possible to the physics described by the IAM amplitude, we have also considered the finite- $T$ contribution from tadpole terms, which becomes relevant as the $\sigma$ meson pole is driven towards the two-pion threshold and its behavior is no longer dominated by the two-pion phase space [47]. The corresponding diagrams are depicted in Fig. 4.2 and, to lowest order in the chiral counting, they emerge from $\mathcal{O}\left(p^{2}\right)$ interaction terms with up to six meson fields. The finite- $T$ correction to the tree-level amplitude to account for these terms reads

$\delta V_{\text {tad }}^{T}(s)=\left[\frac{20}{9} s I_{0}^{T}-\frac{25}{6} I_{2}^{T}\right] / f_{\pi}^{4}+V_{\text {on }}(s) \frac{4}{3 f_{\pi}^{2}} I_{0}^{T}$,

with $I_{2}^{T}=m_{\pi}^{2} I_{0}^{T}$.

In Fig. 4.3 (left) we show the $\sigma$ pole trajectory for the thermal calculation in the BS equation approach with the $\mathcal{O}\left(p^{2}\right)$ kernel including the thermal corrections discussed above. As we can see, the evolution of the pole follows quite

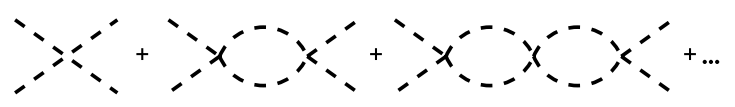

Fig. 4.1 Diagrammatic representation of the Bethe-Salpeter equation for $\pi \pi$ scattering

Fig. 4.2 Pion tadpole diagrams in $\pi \pi$ scattering

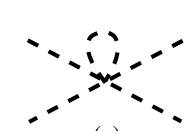

(a)

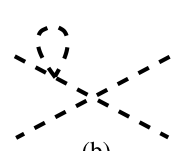

(b) 


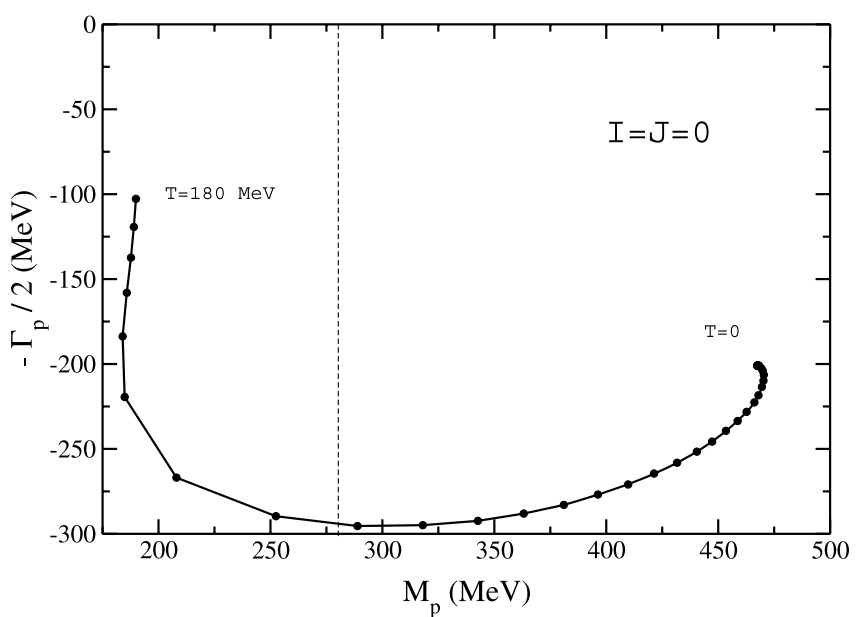

Fig. 4.3 Left: Temperature dependence of the $\sigma$ complex pole in the BS equation approach including thermal corrections to the $\mathcal{O}\left(p^{2}\right)$ kernel (the temperature interval between each point is $5 \mathrm{MeV}$ ). Right:

a similar pattern as in the IAM, although the position of the $\sigma$ mass reaches values further below the two-pion threshold for high temperatures. As in the IAM, the $\sigma$ pole stays far from the real axis, indicating a substantial width for temperatures up to about $200 \mathrm{MeV}$, despite the low value of $M_{\mathrm{p}}$. The stronger attraction felt by the $\sigma$ meson in this approach as compared to the IAM seems to reflect that missing thermal contributions from $t$ - and $u$-channel dynamics are relevant in this energy region and provide a repulsive contribution. Nevertheless, we obtain a qualitatively similar physical behavior of the $\sigma$ pole in both the BS equation approach and the IAM. Thus, we shall use the former as a starting point to incorporate finite-density effects. As a further test, we have also calculated the $\sigma$ pole trajectory in the effective " $f_{\pi}$ scaling" scenario, which we depict in the right-hand panel of Fig. 4.3. We obtain a similar result as in the IAM regarding the $\sigma$ pole collapsing onto the real energy axis for $f_{\pi}$ values below approximately $50 \mathrm{MeV}$. We also find a pole-doubling effect which follows the "molecular" pattern discussed in Sect. 3. Differences are observed at the numerical level as the close-to-threshold behavior of the $\sigma$ pole is dictated by the relative weight of the $\mathcal{O}\left(p^{2}\right)$ and $\mathcal{O}\left(p^{4}\right)$ amplitudes, which are different for the two approaches discussed in this work. Finally, we have also studied threshold enhancement in the $\pi \pi$ amplitude for decreasing values of $f_{\pi}$, which we omit here as our results resemble very much those depicted in Figs. 3.3 and 3.4 for the IAM calculation.

The introduction of nuclear-density effects on top of the temperature follows by a renormalization of the pion propagator in the hot and dense medium. In cold nuclear matter, the pion spectral function exhibits a mixture of the pion quasi-particle mode and $p$-wave particle-hole $(p h)$, Deltahole $(\Delta h)$ excitations [64]. The lowest-order, irreducible $p$ -

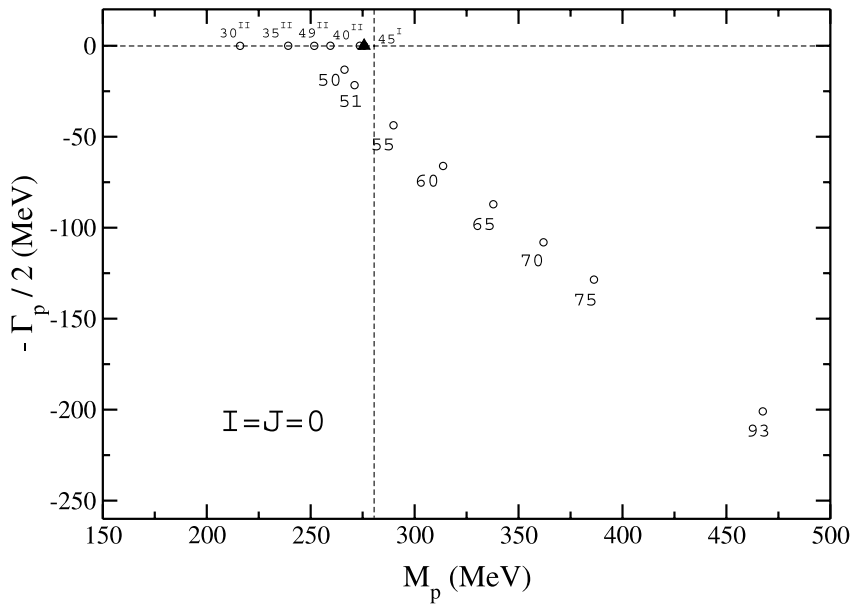

Pole trajectory from $f_{\pi}$ scaling. The numbers attached to each point indicate the value of $f_{\pi}$ in $\mathrm{MeV}$

wave pion self-energy due to $p h$ and $\Delta h$ excitations reads

$$
\begin{aligned}
& \Pi_{\pi N N^{-1}+\pi \Delta N^{-1}}^{p}\left(q_{0}, \boldsymbol{q} ; T\right) \\
& \quad=\left(\frac{f_{N}}{m_{\pi}}\right)^{2} \boldsymbol{q}^{2}\left[U_{N N^{-1}}\left(q_{0}, \boldsymbol{q} ; T\right)+U_{\Delta N^{-1}}\left(q_{0}, \boldsymbol{q} ; T\right)\right],
\end{aligned}
$$

where $U$ stands for the finite-temperature Lindhard function, which we evaluate in imaginary time formalism (ITF) [38, 65], and the density dependence enters $U$ through the baryon chemical potential. We use phenomenological $\pi N N$ and $\pi N \Delta$ coupling constants determined from analysis of pion-nucleon and pion-nucleus reactions, $f_{N} / m_{\pi}=$ $0.007244 \mathrm{MeV}^{-1}$ and $f_{\Delta} / f_{N}=2.13$. The strength of the considered collective modes is modified by repulsive, spinisospin $N N$ and $N \Delta$ short range correlations [64], which we include in a phenomenological way with a single LandauMigdal interaction parameter, $g^{\prime}=0.7$. The RPA-summed (retarded) pion self-energy then reads

$$
\begin{aligned}
& \Pi_{\pi}^{p}\left(q_{0}, \boldsymbol{q} ; T\right) \\
& \quad=\frac{\left(\frac{f_{N}}{m_{\pi}}\right)^{2} F_{\pi}\left(\boldsymbol{q}^{2}\right) \boldsymbol{q}^{2}\left[U_{N N^{-1}}\left(q_{0}, \boldsymbol{q} ; T\right)+U_{\Delta N^{-1}}\left(q_{0}, \boldsymbol{q} ; T\right)\right]}{1-\left(\frac{f_{N}}{m_{\pi}}\right)^{2} g^{\prime}\left[U_{N N^{-1}}\left(q_{0}, \boldsymbol{q} ; T\right)+U_{\Delta N^{-1}}\left(q_{0}, \boldsymbol{q} ; T\right)\right]},
\end{aligned}
$$

where we have accounted for the finite size of $\pi N N$ and $\pi N \Delta$ vertices with the hadronic monopole form factors $F_{\pi}\left(\boldsymbol{q}^{2}\right)=\Lambda_{\pi}^{2} /\left(\Lambda_{\pi}^{2}-\boldsymbol{q}^{2}\right)$, with $\Lambda_{\pi}=1300 \mathrm{MeV}$.

The in-medium pion propagator modifies the analytical structure of the meson-meson loop function, $G$. At lowest order in a density expansion (number of baryon-hole irreducible insertions) the in-medium $\pi \pi$ amplitude at one loop 
Fig. 4.4 Lowest-order baryon-hole insertions in the meson-meson loop function including vertex corrections

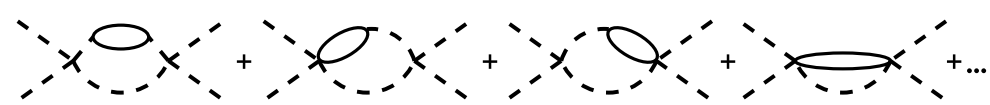

(a)

(b)

(d) includes diagram (a) in Fig. 4.4, in which one of the intermediate pions excites a $p h$ (or $\Delta h$ ) bubble (and similarly with the lower pion line), on top of the vacuum $\pi \pi$ loop (Fig. 4.4, second diagram). Again one is obliged to check whether the on-shell factorization of $V$ and $T$ is still valid in a nuclear medium. This is actually the case for $\pi \pi$ scattering in the scalar channel as it was shown in [26]. The argument is as follows: in addition to diagram (a), chiral symmetry requires a set of meson-baryon contact terms, depicted as diagrams (b-d) (which can be seen as contributions to the $\pi N \rightarrow \pi \pi N$ amplitude by cutting simultaneously the $p h$ bubble and the lower pion line in diagrams (a-d)). It turns out that the contribution from the off-shell part of $V$ in diagram (a) exactly cancels with the sum of the amplitudes from diagrams (b-d), leaving us with diagram (a) with each of the $\pi \pi$ vertices factorized on-shell. Therefore, the algebraic solution of (4.1) is no altered and one only has to replace the vacuum pion propagators in $G$, cf. (4.2), by the inmedium ones, $D_{\pi}=\left[\left(q^{0}\right)^{2}-\omega_{q}-\Pi_{\pi}\right]^{-1}$. The argument holds at finite temperature as we have checked explicitly, in ITF, that the same cancellation of off-shell terms takes place in the thermal amplitude.

In order to re-evaluate $G$ in the hot and dense medium we use the spectral (Lehmann) representation of the pion propagator. The final expression for $G$, once continued onto the real energy axis, reads

$$
\begin{aligned}
& G(P ; \rho, T) \\
& \quad=\int_{0}^{\infty} \frac{\mathrm{d} \Omega}{2 \pi}\left[\frac{1}{P^{0}-\Omega+\mathrm{i} \varepsilon}-\frac{1}{P^{0}+\Omega+\mathrm{i} \varepsilon}\right] F(\Omega),
\end{aligned}
$$

with

$$
\begin{aligned}
F(\Omega)= & \int \frac{\mathrm{d}^{3} q}{(2 \pi)^{3}} \int_{-\Omega}^{\Omega} \mathrm{d} u \pi\left[1-n_{\mathrm{B}}\left(E_{+}\right)-n_{\mathrm{B}}\left(E_{-}\right)\right] \\
& \times S_{\pi}\left(E_{+}, \boldsymbol{q}\right) S_{\pi}\left(E_{-}, \boldsymbol{q}\right),
\end{aligned}
$$

where $S_{\pi}=-\pi^{-1} \operatorname{Im} D_{\pi}$ is the spectral function of the retarded pion propagator and $E_{ \pm}=(\Omega \pm u) / 2$ (we have omitted here the contribution from diffusion poles which typically provides a small correction in the time-like region). Note that $\operatorname{Im} G\left(P^{0}\right)=-F\left(P^{0}\right) / 2$ and thus $F$ plays the role of a generalized in-medium two-pion phase space including both temperature and nuclear-density effects. In the pure thermal case, $F(\sqrt{s})=\sigma_{\mathrm{T}}(s) / 8 \pi \theta\left(s-4 m_{\pi}^{2}\right)$.

In Fig. 4.5 we show the phase-space function, $F\left(P^{0}\right)$, at normal nuclear-matter density $\left(\rho_{0}=0.17 \mathrm{fm}^{-3}\right)$ and different temperatures in the range $T=0-150 \mathrm{MeV}$. We have

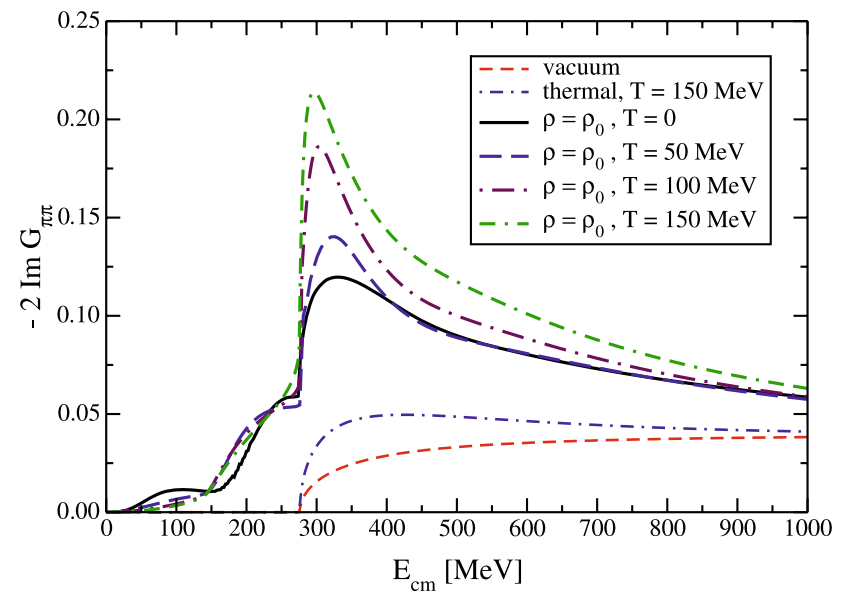

Fig. 4.5 Two-pion phase-space function at finite nuclear density and temperature

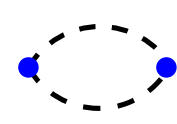

$\mathrm{O}(0)$

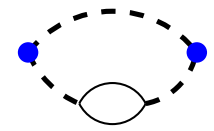

$O(\rho)$

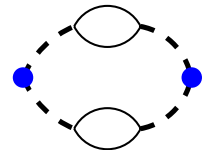

$\mathrm{O}\left(\rho^{2}\right)$
Fig. 4.6 $\mathcal{O}\left(1, \rho, \rho^{2}\right)$ contributions to the meson-meson loop function from irreducible pion self-energy insertions

also depicted the vacuum and thermal cases for comparison. The very first difference that one observes at finite nuclear density is the appearance of strength below the twopion threshold, which is absent in the thermal case. It can be understood on the basis of the baryon-related interaction mechanisms of the pion discussed above. At small nuclear densities, one of the intermediate pions may excite a $p h$ pair whereas the other is placed on the mass shell (see the second diagram in Fig. 4.6). This mechanism is responsible for the strength right below $2 m_{\pi}$ and lowers the threshold down to $m_{\pi}$. For increasing density, the probability for the two pions to be absorbed by baryon-hole excitations sets in (third diagram in Fig. 4.6), which builds additional strength below $m_{\pi}$ and shifts the threshold practically down to $P^{0}=0$ (note the smaller size of this $\mathcal{O}\left(\rho^{2}\right)$ contribution with respect to the excitation of one single $p h$ bubble). Beyond $P^{0}=2 m_{\pi}$, $F\left(P^{0}\right)$ also exhibits a remarkable enhancement with respect to the vacuum and thermal cases, indicating an increased phase space for $\sigma \rightarrow \pi \pi$ decays. This reflects the widely spread structure of the spectral function of the pion in the medium and the considerable attraction experienced by the pion quasiparticle peak. 
When the temperature is increased, the low energy region loses some strength, as the phase space for $N N^{-1}$ excitations is smeared off by the thermal motion of the nucleons. Above $P^{0}=m_{\pi}$, Bose enhancement is more effective as one of the pions is placed on-shell and one can appreciate some increase over the $T=0$ result. The latter effect is strongly magnified right above the two-pion threshold, since (i) the pion spectral function is populated at low energies and (ii) the pion quasiparticle peak is strongly attracted in the medium. Note that the rapid energy dependence of the $p$-wave pion self-energy is also responsible from the quick increase of phase space right beyond the opening of the $\pi \pi$ channel.

We finally show in Fig. 4.7 the imaginary part of the $\pi \pi$ amplitude for different densities and temperatures, which we can compare to the thermal calculation and the " $f_{\pi}$ scaling" scenario, in order to study threshold enhancement effects. We recall here that in the vacuum and thermal cases $\operatorname{Im} T$ vanishes below the two-pion threshold. Our result, from a dynamical calculation of many-body effects in the intermediate two-pion state, exhibits a sizable accumulation of strength at and below $P^{0}=2 m_{\pi}$ already at nuclearmatter density and zero temperature. On the other hand, the amplitude is strongly depleted beyond $\sqrt{s}=400 \mathrm{MeV}$ as compared to the vacuum case, an effect that is not shared by the reduced- $f_{\pi}$ calculation at the equivalent density (cf. Fig. 3.4) which does not account for baryon-related $\sigma$ decay mechanisms. Only for much smaller values of $f_{\pi}$ the strength focuses at the vacuum threshold, as the $\sigma$ pole lies very close to the real energy axis; the resulting enhancement in the amplitude in that case is by far larger than obtained here, which is related to, but not fully driven by the $\sigma$ pole behavior as we explain below. With respect to the thermal calculation, finite density additionally softens the amplitude at high energies and brings strength below the vacuum threshold, a feature which is linked to the presence of a baryonic medium and the excitation of pionic collective modes, as discussed above.

We have also studied the behavior of the $\sigma$ pole at finite nuclear density. The analytic continuation of $T$ in this case is not trivial and we have used an approximated prescription, namely to add to the evaluation of $G$ in the first Riemann sheet the discontinuity on the real axis with respect to the unphysical (second) sheet, Disc $G\left(P^{0}\right)=-2 \mathrm{i} \operatorname{Im} G\left(P^{0}\right)$. Still, this procedure provides the qualitative in-medium behavior of the $\sigma$ pole [29], although its absolute position in the complex plane carries some uncertainty (which we believe is superseded by other theoretical uncertainties in the evaluation of the pion self-energy). The combined effects of temperature and nuclear density accelerate the migration of the $\sigma$ pole towards the $\pi \pi$ threshold. For instance, at $\rho=\rho_{0} / 2$, the $\sigma$ mass reaches $M_{\mathrm{p}}=2 m_{\pi}$ at about $T \simeq 150 \mathrm{MeV}$, whereas at normal nuclear density $M_{\mathrm{p}} \simeq 300 \mathrm{MeV}$ already at $T=0$ and it quickly reaches the two-pion threshold at about $T=100 \mathrm{MeV}$. This is correlated with the cusp structures observed in the amplitude at threshold; cf. Fig. 4.7. However, the strength observed at lower energies is linked to the many-body pion dynamics. In spite of this, the $\sigma$ remains as a broad resonance at nuclear matter density and temperatures approaching the transition one, similarly to what happens in the thermal calculation (at comparatively higher temperatures) and at variance with the simplified " $f_{\pi}$ scaling" approximation. In fact, if we keep increasing the density, at some point the $\sigma$ pole crosses below $2 m_{\pi}$ (one should not trust our implementation of medium effects far beyond $\rho=\rho_{0}$, but as an exercise it provides information about the phase-space behavior of the resonance). For instance, at $\rho=2 \rho_{0}$ and $T=100 \mathrm{MeV}$ the $\sigma$ mass from the pole lies about $70 \mathrm{MeV}$ below the two-pion threshold but still we find $\Gamma_{\mathrm{p}} \simeq 150 \mathrm{MeV}$. A more detailed investigation of
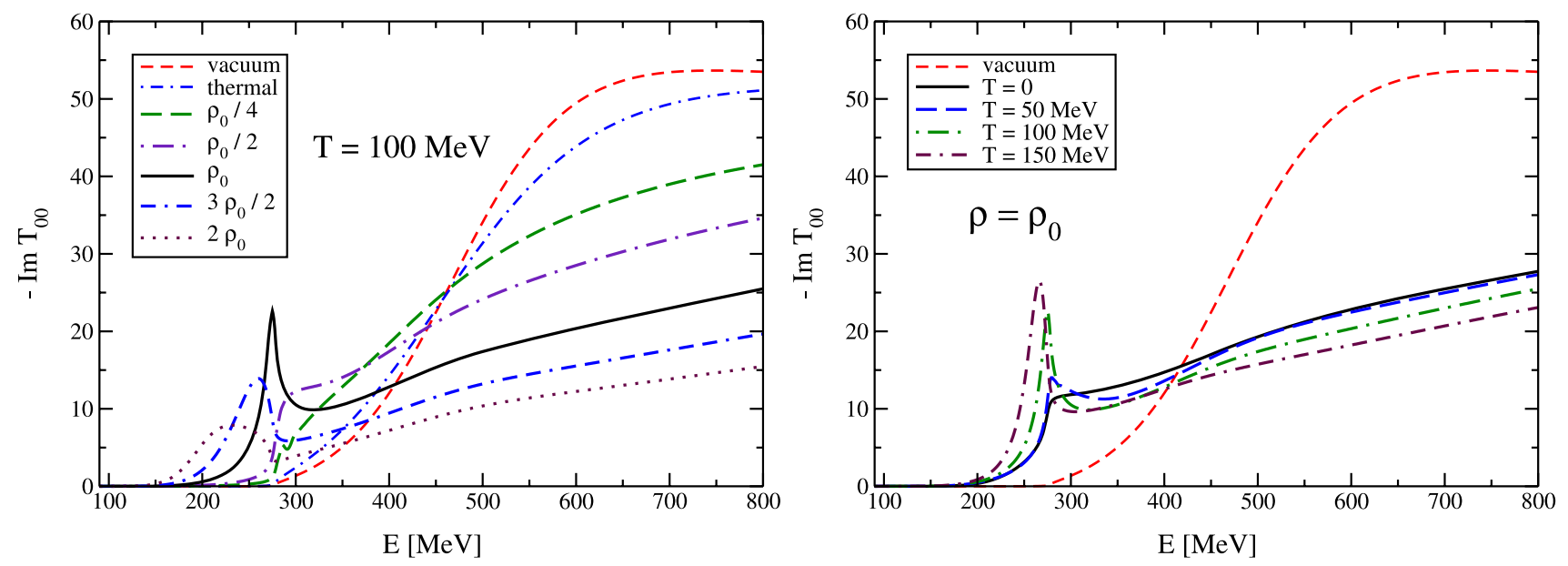

Fig. 4.7 Imaginary part of the $\pi \pi$ amplitude in the $I=J=0$ channel at finite temperature and nuclear density. We also include the result in free space for reference 
possible pole-doubling effects in this approach is on-going and will be reported in a future work.

\section{Conclusions}

We have presented an analysis of the behavior of $\pi \pi$ scattering amplitudes in unitarized chiral perturbation theory with medium effects incorporated in several ways. In particular, we have focused on the behavior with finite temperature and nuclear density of the $\rho(770)$ and $f_{0}(600) / \sigma$ resonances, which are generated dynamically within the inverseamplitude method (IAM).

By considering only thermal effects on the IAM $\pi \pi$ amplitudes, the $\rho$ exhibits a considerable broadening with a small mass decrease as temperature increases, whereas the $\sigma$ mainly decreases its mass, effectively signaling chiralsymmetry restoration, although it still remains as a broad resonance even at the transition temperature. The broadening obtained in our approach for the $\rho$ meson at finite temperature is compatible with the spectral function analysis from dilepton spectra in the recent experiment by the NA60 Collaboration. The evolution of the $\rho$ mass with temperature does not scale as the condensate, which renders our results in quantitative disagreement with the Brown-Rho scaling scenario. The fact that the $\sigma$ pole remains far from the real axis even at the two-pion threshold when only temperature effects are considered implies no significant threshold enhancement for the scattering amplitude, which has been advocated as a precursor of chiral-symmetry restoration. We neither observe a scaling of the $\sigma$ mass with the quark condensate, which indicates that the $f_{0}(600) / \sigma$ resonance dynamically generated in our unitarized chiral approach has a non- $\bar{q} q$ component, which is relevant near the phase transition.

By introducing a finite nuclear density the picture changes dramatically. In a first approximation we have incorporated the effect of a nuclear medium by decreasing $f_{\pi}$ according to the GOR relation to linear order in density. At sufficiently low (high) values of $f_{\pi}$ (density), the $\rho$ and $\sigma$ poles collapse onto the real energy axis at the threshold energy, which is preceded by a significant threshold enhancement in the scattering amplitudes. We have discussed these effects in the context of recent results from resonance production in finite nuclei, and our results are in line with the experimental observations. A detailed analysis reveals that when the resonance pole is close to the real axis it splits into two states in separated Riemann sheets. The markedly different properties of these double poles for the $\rho$ and $\sigma$ channels allows us to classify these $\pi \pi$ resonances according to their internal structure: whereas the $\rho$ meson presents a clear predominant $\bar{q} q$ behavior for high densities (the two poles stay close to threshold, one of them migrating to the first Riemann sheet as a $\pi \pi$ bound state), the $\sigma$ exhibits a "molecular" behavior (one of the poles stays close to threshold, well separated from the other one which evolves to lower energies to become degenerate with the pion). The mass scaling from the $\sigma$ and $\rho$ pole with $f_{\pi}(\rho)$ follows the quark-condensate evolution, and therefore is compatible with a Brown-Rho scaling scenario, although one should keep in mind that relevant finite-density mechanisms are neglected in this approximation.

Finally, we have improved our implementation of finite nuclear-density (and temperature) effects by considering a microscopic calculation of many-body pion dynamics in $\pi \pi$ scattering. We have chosen a different unitarization scheme for the $\pi \pi$ scattering amplitude, namely to solve the BetheSalpeter equation for the lowest-order ChPT interaction. Despite differences in the amplitudes at $\mathcal{O}\left(p^{4}\right)$, this scheme essentially provides the same results as the IAM and allows for a systematic analysis and resummation of a relevant class of pion-interaction mechanisms with the nuclear medium. The pion interactions with the medium are encoded in the single-particle pion self-energy, which accounts for the excitation of $p$-wave particle-hole and Delta-hole components as well as short-distance correlation effects from nucleon-nucleon and Delta-nucleon interactions. The opening of baryon-related channels on top of $\sigma \rightarrow \pi \pi$ at finite density extends the available phase space to lower energies, and therefore the $\pi \pi$ scattering amplitude exhibits an increased strength at and below the two-pion threshold, which is magnified at finite temperature as a consequence of Bose enhancement on the low-energy modes of the $\pi \pi$ intermediate states. Such an effect has been found to provide a satisfactory description of the data from the two-pion photoproduction reaction in nuclei when comparing the mass spectrum in the neutral versus charged-pion channels for different nuclei [25, 29], where nuclear densities of the order of $\rho_{0}$ and below are explored. In our analysis we have considered both finite temperature and nuclear density, thus extending the applicability of the present approach to other experimental scenarios such as the forthcoming heavy-ion physics program at FAIR. As compared to the purely thermal calculation, the attractive-interaction mechanisms of the pion at finite density accelerate the migration of the $\sigma$ pole towards the two-pion threshold. The threshold enhancement observed in the $\pi \pi$ amplitude is correlated to the evolution of the $\sigma$ pole towards (and below) $2 m_{\pi}$. However, differently from the reduced- $f_{\pi}$ result, the $\sigma$ pole stays far from the real axis indicating a sizable decay width of the resonance at densities as high as $2 \rho_{0}$ and temperatures close to the transition one.

As a continuation of this work we plan to implement a similar many-body analysis of finite-density effects for the electromagnetic pion vector form factor, an extension of the present work to the $\mathrm{SU}(3)$ case, where other resonances and 
heavier meson states come into play, and to introduce the effect of a finite meson-number chemical potential. We shall report on these studies elsewhere.

Acknowledgements We acknowledge financial support from Spanish research projects FPA2004-02602, FPA2005-02327, PR34/071856-BSCH, UCM-CAM 910309/08, FPA2007-29115-E and from the F.P.I. Programme (BES-2005-6726). D.C. wishes to thank support from the "Juan de la Cierva" Programme (Ministerio de Educación y Ciencia, Spain).

\section{References}

1. G. Agakichiev et al. (CERES Collaboration), Eur. Phys. J. C 41, 475 (2005)

2. R. Arnaldi et al. (NA60 Collaboration), Phys. Rev. Lett. 96, 162302 (2006)

3. G.E. Brown, M. Rho, Phys. Rev. Lett. 66, 2720 (1991)

4. M. Harada, C. Sasaki, Phys. Rev. D 74, 114006 (2006)

5. M. Herrmann, B.L. Friman, W. Noerenberg, Nucl. Phys. A 545, 267C (1992)

6. R.D. Pisarski, Phys. Rev. D 52, 3773 (1995)

7. W. Peters, M. Post, H. Lenske, S. Leupold, U. Mosel, Nucl. Phys. A 632, 109 (1998)

8. R. Rapp, G. Chanfray, J. Wambach, Nucl. Phys. A 617, 472 (1997)

9. R. Rapp, J. Wambach, Adv. Nucl. Phys. 25, 1 (2000)

10. D. Cabrera, E. Oset, M.J. Vicente Vacas, Nucl. Phys. A 705, 90 (2002)

11. A. Dobado, A. Gomez Nicola, F.J. Llanes-Estrada, J.R. Pelaez, Phys. Rev. C 66, 055201 (2002)

12. D. Fernandez-Fraile, A. Gomez Nicola, E.T. Herruzo, Phys. Rev. D 76, 085020 (2007)

13. J. Adams et al. (STAR Collaboration), Phys. Rev. Lett. 92, 092301 (2004)

14. M. Naruki et al., Phys. Rev. Lett. 96, 092301 (2006)

15. G.E. Brown, M. Rho, Phys. Rep. 363, 85 (2002)

16. T. Hatsuda, S.H. Lee, H. Shiomi, Phys. Rev. C 52, 3364 (1995), arXiv:nucl-th/9505005

17. R. Nasseripour et al. (CLAS Collaboration), Phys. Rev. Lett. 99, 262302 (2007)

18. M. Urban, M. Buballa, R. Rapp, J. Wambach, Nucl. Phys. A 641, 433 (1998), arXiv:nucl-th/9806030

19. T. Hatsuda, T. Kunihiro, Phys. Rev. Lett. 55, 158 (1985)

20. W.M. Yao et al. (Particle Data Group), J. Phys. G 33, 1 (2006)

21. M.G. Alford, R.L. Jaffe, Nucl. Phys. B 578, 367 (2000)

22. J.R. Pelaez, Phys. Rev. Lett. 92, 102001 (2004)

23. F. Bonutti et al. (CHAOS Collaboration), Nucl. Phys. A 677, 213 (2000)

24. A. Starostin et al. (Crystal Ball Collaboration), Phys. Rev. Lett. 85, 5539 (2000)

25. J.G. Messchendorp et al., Phys. Rev. Lett. 89, 222302 (2002)

26. H.C. Chiang, E. Oset, M.J. Vicente-Vacas, Nucl. Phys. A 644, 77 (1998), arXiv:nucl-th/9712047

27. D. Davesne, Y.J. Zhang, G. Chanfray, Phys. Rev. C 62, 024604 (2000)
28. D. Jido, T. Hatsuda, T. Kunihiro, Phys. Rev. D 63, 011901(R) (2000)

29. L. Roca, E. Oset, M.J. Vicente Vacas, Phys. Lett. B 541, 77 (2002)

30. A. Patkos, Z. Szep, P. Szepfalusy, Phys. Rev. D 68, 047701 (2003)

31. D. Cabrera, E. Oset, M.J. Vicente Vacas, Phys. Rev. C 72, 025207 (2005)

32. Y. Hidaka, O. Morimatsu, T. Nishikawa, M. Ohtani, Phys. Rev. D 70, 076001 (2004)

33. P. Schuck, W. Norenberg, G. Chanfray, Z. Phys. A 330, 119 (1988)

34. S. Weinberg, Physica A 96, 327 (1979)

35. J. Gasser, H. Leutwyler, Ann. Phys. 158, 142 (1984)

36. V. Bernard, N. Kaiser, U.G. Meissner, Int. J. Mod. Phys. E 4, 193 (1995)

37. A. Ramos, E. Oset, Nucl. Phys. A 671, 481 (2000)

38. L. Tolos, D. Cabrera, A. Ramos, arXiv:0807.2947 [nucl-th]

39. A. Dobado, M.J. Herrero, T.N. Truong, Phys. Lett. B 235, 134 (1990)

40. A. Dobado, J.R. Peláez, Phys. Rev. D 47, 4883 (1993)

41. A. Dobado, J.R. Peláez, Phys. Rev. D 56, 3057 (1997)

42. J.A. Oller, E. Oset, J.R. Peláez, Phys. Rev. Lett. 80, 3452 (1998)

43. J. Nieves, E. Ruiz Arriola, Phys. Lett. B 455, 30 (1999)

44. J.A. Oller, U.G. Meissner, Phys. Lett. B 500, 263 (2001)

45. A. Gómez Nicola, J.R. Peláez, Phys. Rev. D 65, 054009 (2002)

46. A. Gomez Nicola, J.R. Pelaez, G. Rios, Phys. Rev. D 77, 056006 (2008), arXiv:0712.2763 [hep-ph]

47. A. Gomez Nicola, F.J. Llanes-Estrada, J.R. Pelaez, Phys. Lett. B 550, 55 (2002), arXiv:hep-ph/0203134

48. A. Gómez Nicola, F.J. LLanes-Estrada, J.R. Peláez, Phys. Lett. B 606, 351 (2005)

49. G.E. Brown, M. Rho, arXiv:nucl-th/0509001, nucl-th/0509002

50. A. Dobado, J.R. Peláez, Phys. Rev. D 59, 034004 (1998)

51. M. Gell-Mann, R.J. Oakes, B. Renner, Phys. Rev. 175, 2195 (1998)

52. V. Thorsson, A. Wirzba, Nucl. Phys. A 589, 633 (1995)

53. U.G. Meissner, J.A. Oller, A. Wirzba, Ann. Phys. 297, 27 (2002)

54. G.E. Brown, M. Rho, Phys. Rep. 396, 1 (2004)

55. S. Leupold, U. Mosel, Phys. Rev. C 58, 2939 (1998), arXiv:nuclth/9805024

56. D. Morgan, M.R. Pennington, Phys. Lett. B 258, 444 (1991). [Erratum-ibid. B 269, 477 (1991)]

57. D. Morgan, Nucl. Phys. A 543, 632 (1992)

58. C. Hanhart, J.R. Pelaez, G. Rios, Phys. Rev. Lett. 100, 152001 (2008)

59. J.A. Oller, E. Oset, Nucl. Phys. A 620, 438 (1997). [Erratum-ibid. A 652, 407 (1999)], arXiv:hep-ph/9702314

60. J.A. Oller, E. Oset, J.R. Pelaez, Phys. Rev. D 59, 074001 (1999). [Erratum-ibid. D 60 (1999 ERRAT,D75,099903.2007) 099906], arXiv:hep-ph/9804209

61. J.A. Oller, E. Oset, J.R. Pelaez, Phys. Rev. Lett. 80, 3452 (1998), arXiv:hep-ph/9803242

62. J.A. Oller, E. Oset, Phys. Rev. D 60, 074023 (1999), arXiv:hep-ph/ 9809337

63. J.A. Oller, U.G. Meissner, Phys. Lett. B 500, 263 (2001), arXiv: hep-ph/0011146

64. E. Oset, H. Toki, W. Weise, Phys. Rep. 83, 281 (1982)

65. M. Urban, M. Buballa, R. Rapp, J. Wambach, Nucl. Phys. A 673, 357 (2000), arXiv:nucl-th/9910004 